\title{
BMJ Open Prevalence and trends of thinness, overweight and obesity among children and adolescents aged 3-18 years across Europe: a protocol for a systematic review and meta-analysis
}

\author{
Miriam Garrido-Miguel, ${ }^{1}$ Iván Cavero-Redondo, ${ }^{1}$ Celia Álvarez-Bueno, ${ }^{1}$ \\ Fernando Rodriguez-Artalejo, ${ }^{2}$ Luis Moreno Aznar, ${ }^{3}$ Jonatan R Ruiz, ${ }^{4}$ \\ Vicente Martinez-Vizcaino ${ }^{1,5}$
}

To cite: Garrido-Miguel M, Cavero-Redondo I, ÁlvarezBueno $\mathrm{C}$, et al. Prevalence and trends of thinness, overweight and obesity among children and adolescents aged 3-18 years across Europe: a protocol for a systematic review and meta-analysis. BMJ Open 2017;7:e018241. doi:10.1136/ bmjopen-2017-018241

- Prepublication history and additional material for this paper are available online. To view these files, please visit the journal online (http://dx.doi org/10.1136/bmjopen-2017018241).

Received 16 June 2017 Revised 6 October 2017 Accepted 7 November 2017

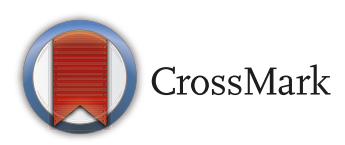

For numbered affiliations see end of article.

Correspondence to Celia Álvarez-Bueno; Celia.Alvarezbueno@uclm.es

\section{ABSTRACT}

Introduction Increasing prevalence of both thinness and excess weight during childhood and adolescence is a significant public health issue because of shortterm health consequences and long-term tracking of weight status. Monitoring weight status in Europe may serve to identify countries and regions where rates of these disorders are either slowing down or increasing to evaluate recent policies aimed at appropriate body weight, and to direct future interventions. This study protocol provides a standardised and transparent methodology to improve estimating trends of thinness, overweight and obesity in children aged 3-18years and adolescents across the European region between 2000 and 2017. Methods and analysis This protocol is guided by the Preferred Reporting Items for Systematic Review and Meta-Analysis Protocols (PRISMA-P) and the Cochrane Collaboration Handbook. To identify relevant studies, a search will be conducted in MEDLINE, EMBASE, Cochrane Library, CINAHL and Web of Science databases. From the selected studies, relevant references will be screened as supplemental sources. Finally, open search in websites from health institutions will be conducted to identify weight status data not published in scientific journals. Cross-sectional, follow-up studies and panel surveys reporting weight status (objectively measured height and weight) according to the International Obesity Task Force criteria, and written in English or Spanish will be included. Subgroup analyses will be carried out by gender, age, study year and country or European region.

Discussion This study will provide a comprehensive description of weight status of children and adolescents across Europe from 2000 to 2017. The results will be disseminated in a peer-reviewed journal. This study will use data exclusively from published research or institutional literature, so institutional ethical approval is not required. PROSPERO registration number CRD42017056917.

\section{INTRODUCTION}

Over the past two decades, the steady increase in overweight and obesity among children and
Strengths and limitations of this study

- This systematic review protocol presents a comprehensive and standardised methodology to identify relevant studies and retrieve information for monitoring trends in thinness, overweight and obesity among children and adolescents across European countries.

- Study selection, data extraction and risk of bias assessment will be carried out independently by two researchers.

- The exclusive use of the International Obesity Task Force criteria will limit comparability with studies using criteria such as those from the Centers for Disease Control or WHO.

- Differences in sample characteristics, geographical location and quality of the included data may increase heterogeneity between studies, which might reduce the quality of evidence on time trends in weight status.

adolescents has become a major public health problem, which has now reached epidemic dimensions in most low/middle-income countries. ${ }^{1-3}$ According to estimates from the WHO Childhood Obesity Surveillance Initiative (COSI), around 33\% European children aged 6 to 9 years were overweight or obese in 2010. ${ }^{4}$ This is a significant increase compared with 2008 , when estimates were $25 \% .^{5}$ Paradoxically, while some projections anticipated that prevalence rates would continue to increase significantly, ${ }^{6}$ previous reviews have shown a plateau, or even a decrease, in prevalence rates of overweight and obesity among children and adolescents in some countries. ${ }^{78}$ Notwithstanding, excess weight in early life will be a persistent worldwide public health problem due to its elevated frequency, and its 
association with physical and mental health disorders in childhood $^{910}$ and adult life. ${ }^{11-13}$

There is also evidence of a growing prevalence of underweight in school-age children in some wealthy countries. ${ }^{14-16}$ Although overweight and thinness in childhood frequently coexist within a single family, the former has been investigated deeply, while the latter has received less attention. This coexistence of weight status disorders, sometimes referred as 'dual burden households', occurs in all countries and is of great relevance because population strategies mainly focus on reducing obesity and may have a negative impact on children with normal or below normal weight. ${ }^{1718}$

Several analyses of excess weight trends among European children and adolescents over the last few decades have rendered somewhat discrepant results. ${ }^{119}$ Also, some studies are relatively outdated, ${ }^{20}$ were based on self-reported weight and height data, ${ }^{20-22}$ and included only a few European countries ${ }^{19}$ or may have limited uncertain population representativeness. ${ }^{23}$ Thus, monitoring both underweight and excess weight trends across European countries using data objectively measured and obtained with comparable methods should be considered a public health priority.

The COSI project ${ }^{45}$ is a valuable effort to obtain population-based data on the prevalence of overweight and obesity among children aged 6 to 9 years from 12 European countries using harmonised surveillance methods. However, we are not aware of any previous study that has summarised the prevalence and trends of both thinness and overweight/obesity in children and adolescents in a wide range of ages from most European countries. Therefore, this novel methodological study protocol was aimed at presenting a clear, standardised and transparent procedure to systematically review, assess and summarise the existing objectively measured information on the latest estimates of European children and adolescent's weight status categories prevalence and trends from 2000 to 2017.

\section{OBJECTIVES}

The purpose of this study protocol is to report a standardised and transparent methodology for conducting a systematic review and meta-analysis aimed to (1) assess the prevalence and trends of thinness, overweight and obesity among children aged 3-18years and adolescents across Europe from 2000 to 2017; and (2) determine if the prevalence and trends of anthropometrics vary according to gender, age, study year and country or European region.

\section{METHODS AND ANALYSIS}

This systematic review and meta-analyses protocol is based on the Preferred Reporting Items for Systematic Review and Meta-Analysis Protocols (PRISMA-P) ${ }^{24}$ and the Cochrane Collaboration Handbook. ${ }^{25}$ The protocol for this review was registered and published on the Prospero database (Registration number: CRD42017056917).

\section{Inclusion/exclusion criteria for study selection}

Studies providing prevalence estimates of thinness (equivalent to $\mathrm{BMI}<18.5 \mathrm{~kg} / \mathrm{m}^{2}$ at 18 years, including thinness grades 1,2 and 3 ); overweight (BMI $\geq 25 \mathrm{~kg} /$ $\left.\mathrm{m}^{2}\right)$ and obesity $\left(\mathrm{BMI} \geq 30 \mathrm{~kg} / \mathrm{m}^{2}\right)$ that meet all of the following criteria will be included: (1) cross-sectional or follow-up studies and panel surveys in which weight and height were objectively measured by trained personnel; (2) studies reporting population-based weight status prevalence estimates according to body mass index (BMI) cut-offs proposed by the International Obesity Task Force criteria (IOTF) ${ }^{26-28}$; (3) studies including population in the age range of 3-18years; and (4) studies written in English or Spanish.

Studies will be excluded from the analyses when they (1) provide self-reported data only; (2) include a sample size less than 100; (3) do not report the sampling method or the sample composition; (4) the target population was a specific population subgroup, such as aboriginal groups, immigrants or those with a narrow socioeconomic status; (5) are not population-based (eg, athletes or children with specific weight-related morbidities); (6) the prevalence of overweight and obesity is not reported separately; and (7) do not use IOTF criteria to determine weight status categories.

\section{Search strategy}

The literature search will be conducted in MEDLINE (via PubMed), EMBASE, Cochrane Library, CINAHL and Web of Science databases from their inception. Study records will be managed using the Mendeley reference manager.

The search terms will include the following terms combined using Boolean operators: (1) population (children, childhood, schooler, schoolchildren, preadolescent, adolescent, school aged, school-aged); (2) outcome (thinness, underweight, obesity, overweight, body composition, body constitution, weight status, anthropometry, (3) study design (prevalence, trend, epidemiology, observational, cross-sectional, longitudinal); and (4) location (Russia, Germany, Turkey, France, UK, Italy, Spain, Ukraine, Poland, Romania, Kazakhstan, Netherlands, Belgium, Greece, Czech Republic, Portugal, Sweden, Hungary, Azerbaijan, Belarus, Austria, Switzerland, Bulgaria, Serbia, Denmark, Finland, Slovakia, Norway, Ireland, Croatia, Bosnia and Herzegovina, Georgia, Moldova, Armenia, Lithuania, Albania, Macedonia, Slovenia, Latvia, Kosovo, Estonia, Cyprus, Montenegro, Luxembourg, Transnistria, Malta, Iceland, Andorra, Liechtenstein, Monaco, San Marino, Vatican city, Europe) (table 1).

Previous reviews and meta-analyses will be scanned for additional references. Also, relevant references included in the selected studies will be screened as supplemental sources. Finally, an open search in national and international institutional public health and health ministry 
Table 1 Search strategy for Medline

\section{Search terms}

\begin{tabular}{|c|c|}
\hline 1. Population & $\begin{array}{l}\text { (Children OR Childhood OR Schooler OR Preadolescent OR Adolescent OR 'School aged' OR } \\
\text { 'School-aged') }\end{array}$ \\
\hline 2. Outcome & $\begin{array}{l}\text { (Thinness OR Underweight OR Obesity OR Overweight OR ‘Body composition' OR ‘Body } \\
\text { constitution' OR ‘Weight status' OR anthropometr^) }\end{array}$ \\
\hline 3. Study design & (Prevalence OR Trend OR Epidemiolog*) \\
\hline 4. Types of studies & (Observant* OR 'cross-sectional' OR longitudinal NOT (survey* OR review)) \\
\hline
\end{tabular}

1 and 2 and 3 and 4 and 5

Truncation symbol: $*=$ =all possible word endings included.

Limits, publication languages: English.

websites will be conducted to identify weight status estimates not reported in scientific journals.

\section{Selection of studies and data extraction}

To identify eligible studies for this systematic review, two reviewers will independently screen titles and abstracts. After excluding those clearly not meeting the selection criteria, the full text of the identified studies will be retrieved and examined. Finally, two reviewers will check the included and excluded studies, and verify the reasons why they were included or excluded. Disagreements will be resolved by consensus; when disagreements persist after discussion, a third reviewer will be consulted. The process of identifying, screening and including or excluding studies will be shown using the PRISMA ${ }^{24}$ flow chart (figure 1).

Two authors will extract data on first author 's name, publication year, study design, period of study, country,
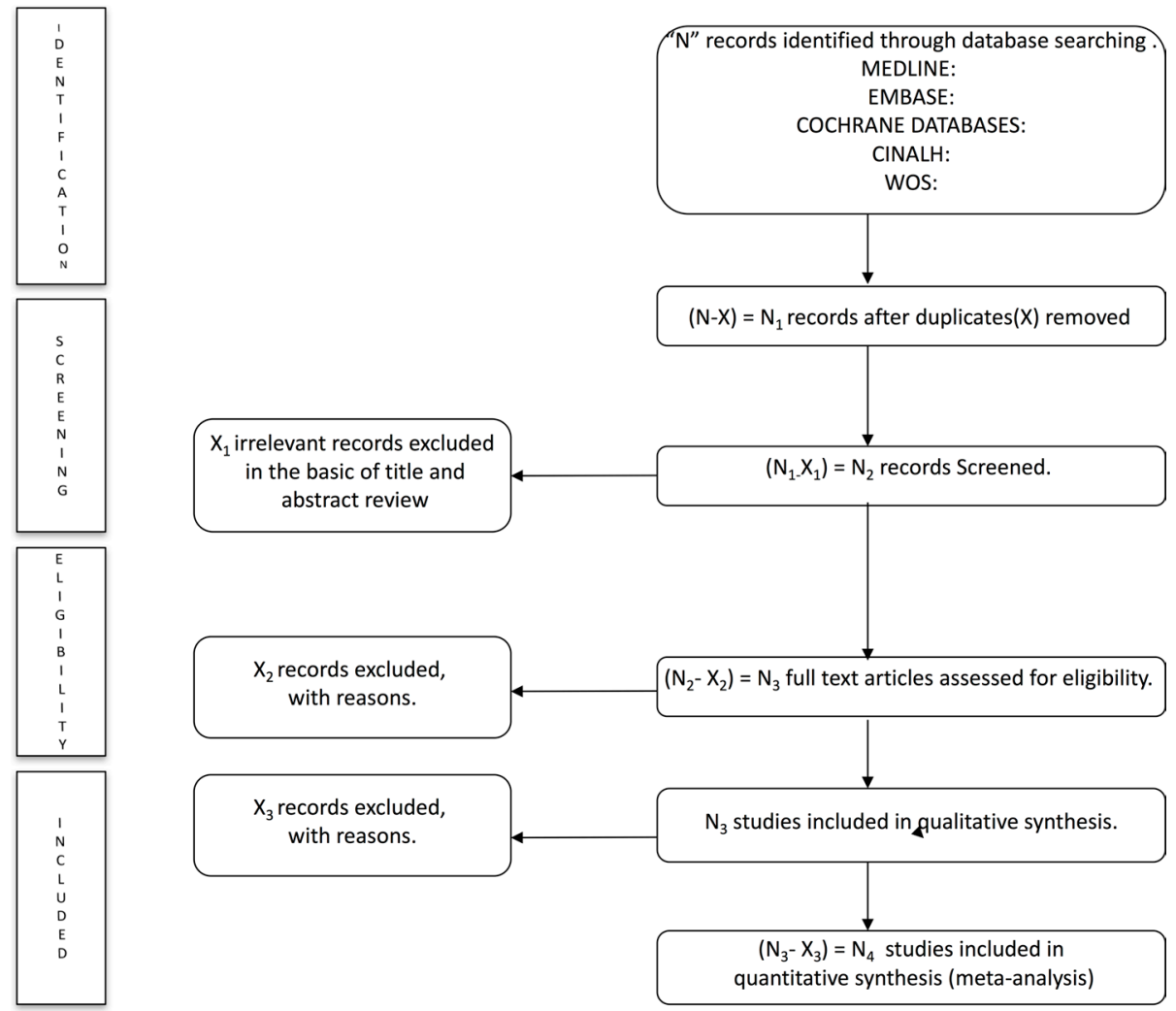

Figure 1 PRISMA (referred Reporting Items for Systematic Review and Meta-Analysis) flow diagram of identification, screening, eligibility and inclusion studies. 
characteristics of the study population (sample size, age of participants, sex) and prevalence of thinness, overweight and obesity. ${ }^{26-28}$ Then, based on the information provided in each paper, the total prevalence for each weight status category will be extracted for the following strata: country or region, age range (3-6, 7-13 and 14-18 years), sex and age-sex combined. Finally, trends in three different time periods (2000-2006, 2007-2010 and 20112017) for each weight category will be assessed.

Any disagreement in data extraction will be resolved by discussion to reach a consensus. When necessary, authors of potentially eligible studies will be contacted to obtain any missing information.

\section{Assessment of risk of bias}

Two reviewers will independently assess risk of bias of each study using the Joanna Briggs Institute tool. ${ }^{29}$ This tool includes 10 criteria that can be assessed as 'yes' $(=1)$, 'no' $(=0)$, 'not applicable' (=NA) or 'unclear' (=?) (online supplementary appendix 1). Thus, the score for each study can range from 0 to 10 . Depending on the number of criteria that each study meets, it can be considered as low risk of bias (7-10), moderate risk of bias (4-6) or high risk of bias (1-3). The Effective Public Health Practice Project ${ }^{30}$ Quality Assessment Tool for Quantitative Studies (online supplementary appendix 2) will be used to assess the quality of longitudinal studies. Studies will be evaluated in seven domains: selection bias, study design, confounders, blinding, data collection method, and withdrawals and drop-outs. Each quality domain could score as strong, moderate or weak, and quality of studies could be classified as strong (with no weak domains), moderate (with one weak domain) and weak (with two or more weak domains). A third reviewer will independently evaluate both assessments and conflicts will be resolved by consensus.

\section{Statistical analysis}

Researchers will summarise the main characteristics of selected studies, including the study's general profile, methods, characteristics of study participants and outcomes in table 2. After data have been extracted, they will determine whether a meta-analysis is possible. At least two observations addressing the same specific outcome will be required to conduct pooled analyses. Data from cross-sectional and longitudinal studies will be used to determine the pooled means of prevalence. For longitudinal studies, data from the beginning and the end of the study will be used as prevalence information sources, without considering reported changes in prevalence. When studies presented estimates of morbid obesity (equivalent to $\mathrm{BMI} \geq 35 \mathrm{~kg} / \mathrm{m}^{2}$ at 18 years), they will be included in the obesity category, in order to estimate an unique valour for obesity (defined as equivalent $\mathrm{BMI} \geq 30 \mathrm{~kg} / \mathrm{m}^{2}$ at 18 years. Finally, when more than one population provides data on the same sample, the one reporting the most detailed results and/or the largest sample size will be retained in data synthesis.

Whenever possible, a meta-analysis will be conducted using STATA V.14 software to combine the pooled mean differences with 95\% CIs. The Mantel-Haenszel fixed-effect method $^{31}$ will be used if there is no evidence of heterogeneity; otherwise, the DerSimonian and Laird random-effects method ${ }^{32}$ will be used.

Heterogeneity among studies will be assessed using the $\mathrm{I}^{2}$ statistic, whose values will be classified as follows: no relevant heterogeneity $(0 \%-40 \%)$, moderate heterogeneity $(30 \%-60 \%)$, substantial heterogeneity $(50 \%-90 \%) \%)$ and considerable heterogeneity $(75 \%-100 \%) \%){ }^{25}$ If there is substantial heterogeneity among studies and a meta-analysis is not possible, a descriptive analysis will be conducted. Finally, publication bias will be evaluated graphically using a funnel plot, as well as with the method proposed by Sterne et $a l^{33}$

\section{Subgroup analysis and meta-regression}

Subgroup and meta-regression analyses will be carried out on the main factors which may cause heterogeneity, such as gender, age (3-6, 7-13 and 14-18 years), country, region (Northern, Central and Southern Europe) and study outcomes (thinness, overweight and obesity) ${ }^{26-28}$ Moreover, to assess prevalence trends, all subgroup analyses will be stratified by three time periods (2000-2006, 2007-2010 and 2011-2017). Additionally, study design, different IOTF criteria, ${ }^{26-28}$ pubertal status based on Tanner score and risk of bias scores ${ }^{25}$ will be considered for additional subgroup analyses.

\section{Sensitivity analysis}

Sensitivity analyses will be conducted excluding studies from the analysis one by one.

\begin{tabular}{|c|c|c|c|c|c|c|c|c|c|}
\hline \multirow[b]{2}{*}{ Reference } & \multirow[b]{2}{*}{$\begin{array}{l}\text { Period of } \\
\text { study }\end{array}$} & \multirow[b]{2}{*}{ Country } & \multirow[b]{2}{*}{$\begin{array}{l}\text { European } \\
\text { region }\end{array}$} & \multicolumn{3}{|c|}{ Population characteristics } & \multicolumn{3}{|l|}{ Outcome } \\
\hline & & & & $\begin{array}{l}\text { Study } \\
\text { design }\end{array}$ & $\begin{array}{l}\text { Age } \\
\text { distribution }\end{array}$ & $\begin{array}{l}\text { Sample } \\
\text { size }\end{array}$ & Underweight (\%) & Overweight (\%) & Obesity (\%) \\
\hline $\begin{array}{l}\text { Fist } \\
\text { author's } \\
\text { name and } \\
\text { year of } \\
\text { publication }\end{array}$ & $\begin{array}{l}\text { Period } \\
\text { of data } \\
\text { collection }\end{array}$ & Country & $\begin{array}{l}\text { European } \\
\text { region }\end{array}$ & $\begin{array}{l}\text { Design } \\
\text { of the } \\
\text { study }\end{array}$ & $\begin{array}{l}\text { Age range of } \\
\text { participants } \\
\text { (years) }\end{array}$ & $\begin{array}{l}\text { Number of } \\
\text { participants } \\
\text { by sex }\end{array}$ & $\begin{array}{l}\text { Prevalence of } \\
\text { underweight } \\
\text { according to IOTF } \\
\text { criteria by sex }\end{array}$ & $\begin{array}{l}\text { Prevalence } \\
\text { of overweight } \\
\text { according to } \\
\text { IOTF criteria by } \\
\text { sex }\end{array}$ & $\begin{array}{l}\text { Prevalence } \\
\text { of obesity } \\
\text { according to } \\
\text { IOTF criteria } \\
\text { by sex }\end{array}$ \\
\hline
\end{tabular}

IOTF, International Obesity Task Force. 


\section{DISCUSSION}

Measuring the prevalence of childhood and adolescence thinness, overweight and obesity, and monitoring changes over time is important from a population health surveillance perspective, and can be used to assist when developing interventions for prevention and control of the obesity epidemic and the increasing prevalence of thinness. However, few studies have reported changes in rates of weight status categories in European countries using a standardised methodology. Therefore, this systematic review and meta-analysis protocol aims to provide a precise, transparent and generalisable methodology for estimating the prevalence and overtime trends of thinness, overweight and obesity for three age groups (3-6, 7-13 and 14-18 years) across the European region during 2000-2017.

Many health interview surveys include questions on self-reported data to monitor weight trends overtime. ${ }^{33} 34$ However, self-reported data could be limited by certain biases. For example, parental report of weight and height could result in height underreporting, which results in biased (higher) BMI estimates. ${ }^{35}$ Thus, only objectively measured data will be used for this systematic review in order to avoid this source of bias.

Furthermore, sources of heterogeneity, such as different geographical locations, study design and sample characteristics (size, age ranges and gender distribution), will be considered in this review. To evaluate whether these variables could affect heterogeneity, random-effects meta-regression will be used. ${ }^{34}$

Children's obesity and parental socioeconomic status have been consistently worldwide related ${ }^{36}$; thus, abrupt socioeconomic changes such as the financial crisis of 2007 presumably influence negatively on the health and welfare of European families, particularly in some southern European countries such as Greece, Spain or Portugal, in which the effect of this crisis have been more serious. In those countries, it has been estimated that between 2005 and 2010, the proportion of children at risk of poverty increased from $20.6 \%$ to $23.7 \%$, and the proportion of them living in unemployed families from $3.7 \%$ to $11.2 \% \cdot{ }^{35}$ For this reason, we decided to separately analyse these three slightly unbalanced time periods: 2000-2006 (a wealthy period), 2007-2010 (the peak of the financial crisis) and 2011-2017 (the aftermath of the crisis)

IOTF criteria ${ }^{26-28}$ will be used because their cut-offs for children and adolescents are representative of the whole world's population, and will allow for better intercountry comparability during the period 2000-2017 than other international criteria, such as those from the Centers for Disease Control (CDC) ${ }^{37}$ or WHO. ${ }^{38}$ Furthermore, considering that two versions of these IOTF criteria ${ }^{26-28}$ have been published during this period (although with minimal differences in their cut-offs), we will perform subgroup analysis in order to discriminate the potential effect of these differences on prevalence determinations. However, using only IOTF criteria ${ }^{26-28}$ limits comparability with other relevant studies across the world, mainly from the USA, where CDC criteria are common and prevent us from collect data prior to 2000. However, as the main objective of this systematic review and meta-analysis is to assess weight status trends, this will not substantially influence on the trend estimations.

Additional potential limitations of this research are publication bias, information bias, poor statistical analyses, and inadequate reporting of methods and findings of the primary studies. It is important to take into account that these sources of bias will be greater in some regions and countries (eg, high-income countries vs low-income countries). However, it is important to summarise the information available from these reports and publications.

In summary, due to lack of complete and unbiased information about prevalence and trends of thinness, overweight and obesity in European children and adolescents, it seems a priority to conduct a systematic review including children and adolescents over the last two decades in order to provide high-quality evidence regarding this important public health problem. Furthermore, given that the European children and adolescents share multiple geographical and socioeconomical circumstances that make them an epidemiological entity, it seems thoughtful to monitor the weight status trends focusing in this region of the world. Also, it is important to show a new model for presenting studies addressing prevalence and trends of cardiovascular risk factors that could be useful as guidelines for future research of these types of issues. Finally, this study will provide updated and valuable information for policymakers and healthcare providers at national and continental levels in order to monitor the effectiveness of ongoing preventive policies in European children and adolescents.

\section{Author affiliations}

${ }^{1}$ Health and Social Research Center, Universidad de Castilla-La Mancha, Cuenca, Spain

${ }^{2}$ Department of Preventive Medicine and Public Health, School of Medicine, Universidad Autónoma de Madrid/IdiPaz, CIBERESP, and IMDEA-Food Institute. CEI UAM+CSIC, Madrid, Spain

${ }^{3}$ GENUD "Growth, Exercise, NUtrition and Development" Research Group, Faculty of Health Sciences, Universidad de Zaragoza, Spain; Instituto Agroalimentario de Aragón(IA2), Spain; Instituto de Investigación Sanitaria Aragón (IIS Aragón), Spain; Centro delnvestigación Biomédica en Red de Fisiopatología de la Obesidad y Nutrición(CIBERObn), Spain

${ }^{4}$ Department of Physical Education and Sports, Faculty of Sport Sciences, PROmoting FITness and Health through Physical Activity Research Group (PROFITH), University of Granada, Granada, Andalucía, Spain

${ }^{5}$ Facultad de Ciencias de la Salud, Universidad Autónoma de Chile, Talca, Chile

\section{Twitter@CESS_UCLM}

Contributors VM-V and MG-M designed the study. VM-V was the principal investigator and guarantor. VM-V and MG-M were the main coordinators of the study. CA-B, IC-R, FR-A, LMA, JRR and VM-V conducted the study. MG-M, IC-R and $C A-B$ gave statistical and epidemiological support. MG-M wrote the article with the support of CA-B and VM-V. All authors reviewed and approved the final version of the manuscript.

Funding MG-M and CA-B are supported by grants from the Ministerio de Educación, Cultura y Deporte, (FPU15/03847 and FPU13/03137, respectively). IC-R is supported by a grant from the Universidad de Castilla-La Mancha (FPI13/01582).

Competing interests None declared. 
Provenance and peer review Not commissioned; externally peer reviewed.

Open Access This is an Open Access article distributed in accordance with the Creative Commons Attribution Non Commercial (CC BY-NC 4.0) license, which permits others to distribute, remix, adapt, build upon this work non-commercially, and license their derivative works on different terms, provided the original work is properly cited and the use is non-commercial. See: http://creativecommons.org/ licenses/by-nc/4.0/

(C) Article author(s) (or their employer(s) unless otherwise stated in the text of the article) 2017. All rights reserved. No commercial use is permitted unless otherwise expressly granted.

\section{REFERENCES}

1. Ng M, Fleming $\mathrm{T}$, Robinson $\mathrm{M}$, et al. Global, regional, and national prevalence of overweight and obesity in children and adults during 1980-2013: a systematic analysis for the global burden of disease study 2013. Lancet 2014;384:766-81.

2. Wang Y, Lobstein T. Worldwide trends in childhood overweight and obesity. Int J Pediatr Obes 2006;1:11-25.

3. de Onis M, Blössner M, Borghi E. Global prevalence and trends of overweight and obesity among preschool children. Am J Clin Nutr 2010;92:1257-64.

4. Wijnhoven TM, van Raaij JM, Spinelli A, et al. WHO European childhood obesity surveillance initiative: body mass index and level of overweight among 6-9-year-old children from school year 2007/2008 to school year 2009/2010. BMC Public Health 2014;14:806

5. Wijnhoven TM, van Raaij JM, Spinelli A, et al. WHO european childhood obesity surveillance initiative 2008: weight, height and body mass index in 6-9-year-old children. Pediatr Obes 2013;8:79-97.

6. Kelly T, Yang W, Chen CS, et al. Global burden of obesity in 2005 and projections to 2030. Int J Obes 2008;32:1431-7.

7. Rokholm B, Baker JL, Sørensen TI. The levelling off of the obesity epidemic since the year 1999--a review of evidence and perspectives. Obes Rev 2010;11:835-46.

8. Olds T, Maher C, Zumin S, et al. Evidence that the prevalence of childhood overweight is plateauing: data from nine countries. Int $J$ Pediatr Obes 2011;6:342-60.

9. Franks PW, Hanson RL, Knowler WC, et al. Childhood obesity, other cardiovascular risk factors, and premature death. N Engl J Med 2010;362:485-93.

10. Sánchez-López M, Salcedo-Aguilar F, Solera-Martínez M, et al. Physical activity and quality of life in schoolchildren aged 11-13 years of Cuenca, Spain. Scand J Med Sci Sports 2009;19:879-84.

11. Baker JL, Olsen LW, Sørensen TI. Childhood body-mass index and the risk of coronary heart disease in adulthood. N Engl $J$ Med 2007;357:2329-37.

12. Franks PW, Hanson RL, Knowler WC, et al. Childhood obesity, other cardiovascular risk factors, and premature death. N Engl J Med Overseas Ed 2010;362:485-93.

13. Herva A, Laitinen J, Miettunen J, et al. Obesity and depression: results from the longitudinal Northern Finland 1966 birth cohort study. Int J Obes 2006;30:520-7.

14. Martínez-Vizcaíno V, Solera Martínez M, Notario Pacheco B, et al. Trends in excess of weight, underweight and adiposity among Spanish children from 2004 to 2010: the Cuenca Study. Public Health Nutr 2012;15:2170-4.

15. Kolle E, Steene-Johannessen J, Holme I, et al. Secular trends in adiposity in Norwegian 9-year-olds from 1999-2000 to 2005. BMC Public Health 2009;9:389.
16. Franssen SJ, van der Wal MF, Jansen P, et al. (Thinness and overweight in children from amsterdam: a trend analysis and forecast). Ned Tijdschr Geneeskd 2015;159:A8967.

17. Doak CM, Adair LS, Bentley M, et al. The dual burden household and the nutrition transition paradox. Int J Obes 2005;29:129-36.

18. de Ruiter I, Olmedo-Requena R, Sánchez-Cruz JJ, et al. Trends in child obesity and underweight in spain by birth year and age, 1983 to 2011. Rev Esp Cardiol 2017;70:646-55

19. Brug J, van Stralen MM, Te Velde SJ, et al. Differences in weight status and energy-balance related behaviors among schoolchildren across Europe: the ENERGY-project. PLoS One 2012;7:e34742.

20. Martínez JA, Kearney JM, Kafatos A, et al. Variables independently associated with self-reported obesity in the European Union. Public Health Nutr 1999;2:125-33.

21. Gallus S, Lugo A, Murisic B, et al. Overweight and obesity in 16 European countries. Eur J Nutr 2015;54:679-89.

22. Lazzeri G, Rossi S, Kelly C, et al. Trends in thinness prevalence among adolescents in ten European countries and the USA (19982006): a cross-sectional survey. Public Health Nutr 2014;17:2207-15.

23. Peytremann-Bridevaux I, Faeh D, Santos-Eggimann B. Prevalence of overweight and obesity in rural and urban settings of 10 European countries. Prev Med 2007;44:442-6.

24. Moher D, Shamseer L, Clarke M, et al. Preferred reporting items for systematic review and meta-analysis protocols (PRISMA-P) 2015 statement. Syst Rev 2015;4:1.

25. Higgins JP, Thompson SG. Quantifying heterogeneity in a metaanalysis. Stat Med 2002;21:1539-58

26. Cole TJ, Bellizzi MC, Flegal KM, et al. Establishing a standard definition for child overweight and obesity worldwide: international survey. BMJ 2000;320:1240-3.

27. Cole TJ, Flegal KM, Nicholls D, et al. Body mass index cut offs to define thinness in children and adolescents: international survey. BMJ 2007;335:194.

28. Cole TJ, Lobstein T. Extended international (IOTF) body mass index cut-offs for thinness, overweight and obesity. Pediatr Obes 2012;7:284-94

29. Munn Z, Moola S, Riitano D, et al. The development of a critical appraisal tool for use in systematic reviews addressing questions of prevalence. Int J Health Policy Manag 2014;3:123-8.

30. Armijo-Olivo S, Stiles CR, Hagen NA, et al. Assessment of study quality for systematic reviews: a comparison of the cochrane collaboration risk of bias tool and the effective public health practice project quality assessment tool: methodological research. J Eval Clin Pract 2012;18:12-18.

31. Leonard T, Duffy JC. A Bayesian fixed effects analysis of the mantel-haenszel model applied to meta-analysis. Stat Med 2002;21:2295-312.

32. DerSimonian R, Kacker R. Random-effects model for meta-analysis of clinical trials: an update. Contemp Clin Trials 2007;28:105-14.

33. Sterne JA, Egger M, Smith GD. Systematic reviews in health care: investigating and dealing with publication and other biases in metaanalysis. BMJ 2001;323:101-5.

34. Krul AJ, Daanen HA, Choi H. Self-reported and measured weight height and body mass index (BMI) in Italy, the Netherlands and North America. Eur J Public Health 2011;21:414-9.

35. Connor Gorber S, Tremblay M, Moher D, et al. A comparison of direct vs. self-report measures for assessing height, weight and body mass index: a systematic review. Obes Rev 2007;8:307-26.

36. Karanikolos M, Mladovsky P, Cylus J, et al. Financial crisis, austerity, and health in Europe. Lancet 2013;381:1323-31.

37. Kuczmarski RJ, Ogden CL, Grummer-Strawn LM, et al. CDC growth charts: United States. Adv Data 2000:1-27.

38. WHO Multicentre Growth Reference Study Group. WHO child growth standards based on length/height, weight and age. Acta Paediatr Suppl 2006;450:76-85. 